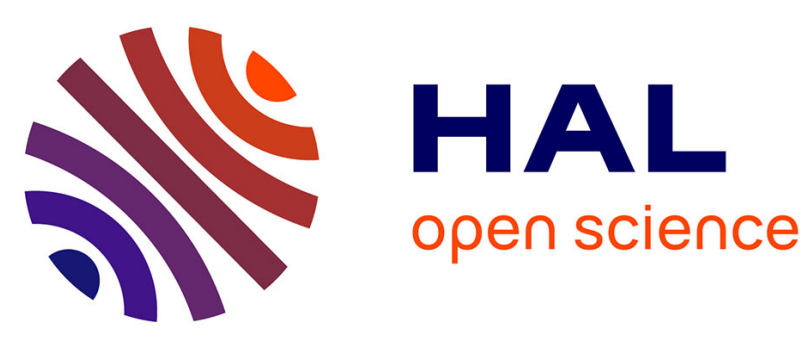

\title{
Langmuir probe in magnetized plasma: Determination of the electron diffusion parameter and of the electron energy distribution function
}

Jean-Louis Jauberteau, Isabelle Jauberteau, O. Daniel Cortázar, Ana Maria Megia-Macias

\section{To cite this version:}

Jean-Louis Jauberteau, Isabelle Jauberteau, O. Daniel Cortázar, Ana Maria Megia-Macias. Langmuir probe in magnetized plasma: Determination of the electron diffusion parameter and of the electron energy distribution function. Contributions to Plasma Physics, 2019, 60 (2), pp.e201900067. 10.1002/ctpp.201900067 . hal-02368547

\section{HAL Id: hal-02368547 \\ https://hal.science/hal-02368547}

Submitted on 8 Dec 2020

HAL is a multi-disciplinary open access archive for the deposit and dissemination of scientific research documents, whether they are published or not. The documents may come from teaching and research institutions in France or abroad, or from public or private research centers.
L'archive ouverte pluridisciplinaire $\mathbf{H A L}$, est destinée au dépôt et à la diffusion de documents scientifiques de niveau recherche, publiés ou non, émanant des établissements d'enseignement et de recherche français ou étrangers, des laboratoires publics ou privés. 


\title{
Langmuir probe in magnetized plasma: Determination of the electron diffusion parameter and of the Electron Energy Distribution Function.
}

\author{
J. L. Jauberteau, ${ }^{1, a}$ I. Jauberteau, ${ }^{1}$ O. D. Cortázar, ${ }^{2}$ and A. Megía-Macías ${ }^{3}$
}

${ }^{1}$ Université de Limoges, UMR 7315 CNRS, SPCTS, 12 rue Atlantis, 87068 Limoges, France

${ }^{2}$ Universidad de Castilla-La Mancha, ETSII-INEI, Av. Camilo Jose Cela s/n, 13071 Ciudad Real, Spain

${ }^{3}$ University of Deusto, Department of Industrial Technologies, Faculty of Engineering, Av. Universidades, 24, 48007 Bilbao, Vizcaya, Spain

aE-mail: jean-louis.jauberteau@unilim.fr

\begin{abstract}
.
This work is devoted to a new method used to determine the electron diffusion parameter and the Electron Energy Distribution Function (EEDF) in magnetized plasma, by means of a Langmuir single probe. This method is only based on calculations performed on experimental data, no model is used and results do not depend on the accuracy of the different hypothesis, the probe orientation within the plasma, the magnetic field intensity, the diffusion coefficient, the mean free path or the potential profile through the sheath. We test the method and show its efficiency with experiments performed in hydrogen magnetized microwave plasma, using magnetic field intensity up to $0.12 \mathrm{~T}$. Results are compared to those obtained using classical methods based on models and hypothesis reported in literature.
\end{abstract}

\section{Introduction.}

In a magnetised gas discharge, the diagnostic by means of Langmuir probes is influenced by the charge particle gyration along the magnetic field. The gyration radius in the magnetic field depends on the mass and charge of the particle and is given by the Larmor radius $\mathrm{R}_{\mathrm{L}}=\mathrm{mv} / \mathrm{qB}$, where $\mathrm{m}, \mathrm{v}, \mathrm{q}$ and $\mathrm{B}$ are the mass, velocity, charge of the particle and the intensity of the magnetic field, respectively. In cold plasma with ion and electron mean energies equal to $0.1 \mathrm{eV}$ and $5 \mathrm{eV}$, respectively the ratio of the ion to electron Larmor radius is 6 for $\mathrm{H}^{+}$ion. In weakly ionized plasma, collisions are mainly ion-neutral and electron neutral collisions and the ion mean free path is about one order of magnitude smaller than the electron mean free 
path. It is much lower than the ion Larmor radius at low magnetic field intensity. So, the ion rotation can be neglected compared to the electron one $[1,2]$.

Because of the electron rotation along the magnetic field, the radial component of the electron diffusion coefficient (perpendicular to the magnetic field) is lower than the longitudinal one $D_{\perp}=\frac{D_{\|}}{\alpha^{2}}$, with $\alpha=\left(1+\left(\frac{\lambda\left(\varepsilon_{e}\right)}{R_{L}}\right)^{2}\right)^{1 / 2}$. It depends on the ratio of the electron mean free path $\left(\lambda\left(\varepsilon_{\mathrm{e}}\right)\right)$ to the Larmor radius.

The longitudinal diffusion coefficient component is given by $D_{/ /}=\frac{1}{3} \lambda\left(\varepsilon_{e}\right) v_{e}$ and depends on the electron mean free path and on the electron mean velocity.

The early work of Swift [3] concerning the effect of the disturbance of the plasma produced by the drain of electron through the sheath around the probe in the case of high pressure plasma has been used and adjusted in the case of magnetized plasma [4,5]. In the case of magnetized plasma, the diffusion coefficient is anisotropic and according to the cylindrical probe orientation in the magnetic field, the flux of electrons collected by the probe changes because of the change of the electron diffusion coefficient value in the flux tube formed around the probe. The electron current collected by the Langmuir probe depends on the probe orientation in the magnetic field and on parameters like the diffusion coefficient or the electron mean free path which change through the sheath formed around the probe.

The electron flow $\mathrm{d} \Phi\left(\varepsilon_{\mathrm{e}}\right)$ of energy ranging from $\varepsilon_{\mathrm{e}}$ and $\varepsilon_{\mathrm{e}}+\mathrm{d} \varepsilon_{\mathrm{e}}$ depends on the diffusion phenomenon and assuming only a radial diffusion through the sheath, it is given by [3],

$d \Phi\left(\varepsilon_{e}\right)=-D \frac{\partial n\left(\varepsilon_{e}\right)}{\partial r}=-D \frac{\partial\left[F\left(\varepsilon_{e}\right) d \varepsilon_{e}\right]}{\partial r}$

Where, $r, F\left(\varepsilon_{e}\right)$ and $D$ are the radius through the sheath, the EEDF and the diffusion coefficient, respectively. So, the change of EEDF due to the diffusion of electrons through the sheath is,

$$
\left[F_{r}\left(\varepsilon_{e}\right)-F_{0}\left(\varepsilon_{e}\right)\right] d \varepsilon_{e}=d j_{e}\left(\varepsilon_{e}\right) \int_{r o}^{r} \frac{d r}{e D S}
$$

Where, $S$ is the collecting probe area, and $\mathrm{dj}_{\mathrm{e}}\left(\varepsilon_{\mathrm{e}}\right)=-\mathrm{eSd} \Phi\left(\varepsilon_{\mathrm{e}}\right)$.

The change of EEDF through the sheath depends on the diffusion coefficient, the larger the diffusion coefficient, the lower the change in EEDF.

Using the Langmuir law to calculate the electron current collected by a probe biased with a retarding potential $\mathrm{V}$, 
$j_{e}(V)=g \int_{e V}^{\infty} \varepsilon_{e}^{1 / 2} F\left(\varepsilon_{e}\right)\left[1-\frac{e V}{\varepsilon_{e}}\right] d \varepsilon_{e}$,

where $g=\frac{e S}{4} \sqrt{\frac{2}{m_{e}}}$ and $\mathrm{V}$ is the difference of potential between the plasma and the bias voltage of the probe and combining $\mathrm{EQ}(2)$ and $\mathrm{EQ}(3)$, we obtain for the electron current collected by the probe in the case of a retarding potential $\mathrm{V}$ [4],

$$
j_{e}(V)=g \int_{e V}^{\infty} \frac{\left(\varepsilon_{e}-e V\right) \varepsilon_{e}^{-1 / 2} F_{0}\left(\varepsilon_{e}\right) d \varepsilon_{e}}{\left[1+\left(\frac{\left(\varepsilon_{e}-e V\right)}{\varepsilon_{e}}\right) \Psi\left(\varepsilon_{e}\right)\right]}
$$

It shows that the electron current collected by the probe negatively bias depends on a parameter $\Psi\left(\varepsilon_{\mathrm{e}}\right)$ which is called the electron diffusion parameter, it depends on the electron diffusion coefficient and because of the magnetic field, it changes with the orientation of the probe in the plasma, the electron energy and the retarding potential applied to the probe. The knowledge of $\Psi\left(\varepsilon_{\mathrm{e}}\right)$ is necessary to determine accurate EEDF from the electron current.

Authors have attempted to model the electron diffusion through the sheath to calculate the diffusion parameter $[4,5,6]$. They consider the probe as an ellipsoïd of revolution and assume a constant diffusion coefficient and electron mean free path through the sheath. Moreover, the diffusion parameter also depends on the hypothesis which are made for the voltage profile through the sheath and on a $\gamma$ factor due to the probe geometry. Consequently, the EEDF calculated using the experimental I-V probe characteristics depends on all hypothesis made to simplify the calculations.

In this work $\Psi\left(\varepsilon_{\mathrm{e}}\right)$ is directly calculated using I-V probe characteristics and no model and hypothesis on the diffusion coefficient, the electron mean free path or the potential profile through the sheath is necessary to calculate the diffusion parameter and the EEDF. We just consider the hypothesis used to obtain EQ3 and EQ4: The EEDF measured is representative of the plasma and is not disturbed by the reactor wall or by the probe collecting surface.

In the next part of this paper we detail our method used to calculate the electron diffusion parameter and EEDF from experimental data. We compare the results with those calculated using the model reported in literature. 
2. Determination of the experimental value of the diffusion parameter and calculation of the EEDF.

As shown in literature $[4,5,6]$, the first derivative of EQ4 versus V is,

$$
\frac{\partial j_{e}(V)}{\partial V}=g \int_{e V}^{\infty} \frac{-e \varepsilon_{e}^{-1 / 2} F_{0}\left(\varepsilon_{e}\right) d \varepsilon_{e}}{\left[1+\left(\frac{\varepsilon_{e}-e V}{\varepsilon_{e}}\right) \Psi\left(\varepsilon_{e}\right)\right]^{2}},
$$

and the second derivative versus $\mathrm{V}$ is,

$\frac{\partial^{2} j_{e}(V)}{\partial V^{2}}=C \varepsilon_{e}^{-1 / 2} F_{0}\left(\varepsilon_{e}\right)-C \int \frac{2 \Psi\left(\varepsilon_{e}\right) \varepsilon_{e}^{3 / 2} F_{0}\left(\varepsilon_{e}\right) d \varepsilon_{e}}{\left[\varepsilon_{e}\left(1+\Psi\left(\varepsilon_{e}\right)\right)-e V \Psi\left(\varepsilon_{e}\right)\right]^{3}}$

$$
C=\frac{e^{3} S}{2 \sqrt{2 m_{e}}}
$$

Using EQ4 we have also,

$$
A=\frac{\partial j_{e}(V)}{\partial \varepsilon_{e}}=\frac{\partial j_{e}(V)}{\partial V} \frac{\partial V}{\partial \varepsilon_{e}}=g \frac{\left(\varepsilon_{e}-e V\right) \varepsilon_{e}^{-1 / 2} F_{0}\left(\varepsilon_{e}\right) d \varepsilon_{e}}{\left[1+\left(\frac{\left(\varepsilon_{e}-e V\right)}{\varepsilon_{e}}\right) \Psi\left(\varepsilon_{e}\right)\right]}
$$

and using EQ5,

$$
B=\frac{\partial\left(\frac{\partial j_{e}(V)}{\partial V}\right)}{\partial \varepsilon_{e}}=\frac{\partial^{2}\left(j_{e}(V)\right)}{\partial V^{2}} \frac{\partial V}{\partial \varepsilon_{e}}=g \frac{-e \varepsilon_{e}^{-1 / 2} F_{0}\left(\varepsilon_{e}\right) d \varepsilon_{e}}{\left[1+\left(\frac{\varepsilon_{e}-e V}{\varepsilon_{e}}\right) \Psi\left(\varepsilon_{e}\right)\right]^{2}}
$$

Thus, $\Psi\left(\varepsilon_{\mathrm{e}}\right)$ can be deduced from the ratio $\mathrm{A} / \mathrm{B}$ and is given by,

$$
\Psi\left(\varepsilon_{e}\right)=-\left[\frac{e}{\left(\varepsilon_{e}-e V\right)} \frac{\frac{\partial j_{e}(V)}{\partial V}}{\frac{\partial^{2} j_{e}(V)}{\partial V^{2}}}+1\right] \frac{\varepsilon_{e}}{\left(\varepsilon_{e}-e V\right)} .
$$


This result shows that the diffusion parameter can be directly determined from experimental data (without any model and assumption) considering the first and second derivative versus $\mathrm{V}$ of the electron current intensity measured by means of electrostatic single probe. This method has been tested on different probe characteristics measured in magnetized plasma with $\mathrm{B}$ ranging from $0 \mathrm{~T}$ to $0.12 \mathrm{~T}$. In the following part, we consider one example to explain how the EEDF can be calculated from the diffusion parameter determined using experimental data and we compare the results with those obtained using the method given in literature $[4,5]$.

The probe characteristic has been measured in the microwave plasma working at $2.45 \mathrm{GHz}$ with a power equal to $1500 \mathrm{~W}$ and sustained in hydrogen at $0.19 \mathrm{~Pa}$. The Langmuir probe is a cylindrical single probe (tungsten wire $6 \mathrm{~mm}$ length and $0.5 \mathrm{~mm}$ diameter), located in the middle of the reactor along the cylindrical reactor axis and is parallel to the magnetic field. Measurements are performed at the steady state, at $103 \mathrm{~mm}$ from the microwave injector. The applied magnetic field intensity is equal to $97 \mathrm{mT}$. A detail of the experimental set up is given in ref [7]. The second derivative of the electron current collected versus retarding applied voltage is calculated using the numerical method that we have developed and already tested on probe characteristics [8,9]. This method is efficient for a noisy signal and no extra averaging is necessary to minimize the noise.

Fig1 shows the current intensity and second derivative of the current intensity versus $\mathrm{V}$ corresponding to the probe characteristic under investigation. The ion current collected at saturation is determined at large negative bias voltage i.e. for $\mathrm{V}_{\text {bias }}$ ranging from -150 to $100 \mathrm{~V}$, when all electrons are repelled. As shown on Fig1, it changes linearly with the applied bias voltage $\left(\mathrm{Ii}(\mathrm{A})=5 \times 10^{-5} \mathrm{~V}-0.0195\right)$. The collected electron current is calculated removing the ion current from the total current.

It is worth noting that in magnetized plasma electron current collected by the single probe tip can be strongly decreased and lower than it is usually measured in plasma without magnetic field. As shown on Fig1, the ratio of the electron current at saturation to the ion current at saturation is about 2 . The reason of this low ratio value measured when the single probe is immersed in magnetized plasma has been largely explained in the literature $[3,4,5,6]$. In magnetized plasma, the charged particles are rotating in the magnetic field. The electron diffusion coefficient is anisotropic and the radial component (perpendicular to the magnetic field) is much lower than the parallel one. When the ion gyration in plasma can be neglected i.e. when the ion Larmor radius is very large compared to the probe radius, the ratio of the electron current at saturation to the ion current at saturation decreases more or less according to the probe orientation with increasing magnetic field. In the present work where single 
probe is used, any electron whatever its energy can be collected depending on the retarding potential applied to the probe and the electron current collected is given by EQ4.

The first derivative of the electron current is calculated integrating the second derivative of the electron current over $\mathrm{V}$ ranging from $\mathrm{V}=0$ to $\mathrm{V}=\mathrm{V}_{\mathrm{p}}-\mathrm{V}_{\text {bias. }}$. Fig2 shows the electron current, the first and second derivative versus the applied voltage. The value of $\Psi\left(\varepsilon_{\mathrm{e}}\right)$ versus $\varepsilon_{\mathrm{e}}$ is determined from these values using EQ9. Results are shown on Fig 3. The diffusion parameter is strongly decreasing with increasing electron energy. This can be ascribed to the Larmor radius which increases with increasing electron energy and to the diffusion coefficient which decreases with decreasing Larmor radius.

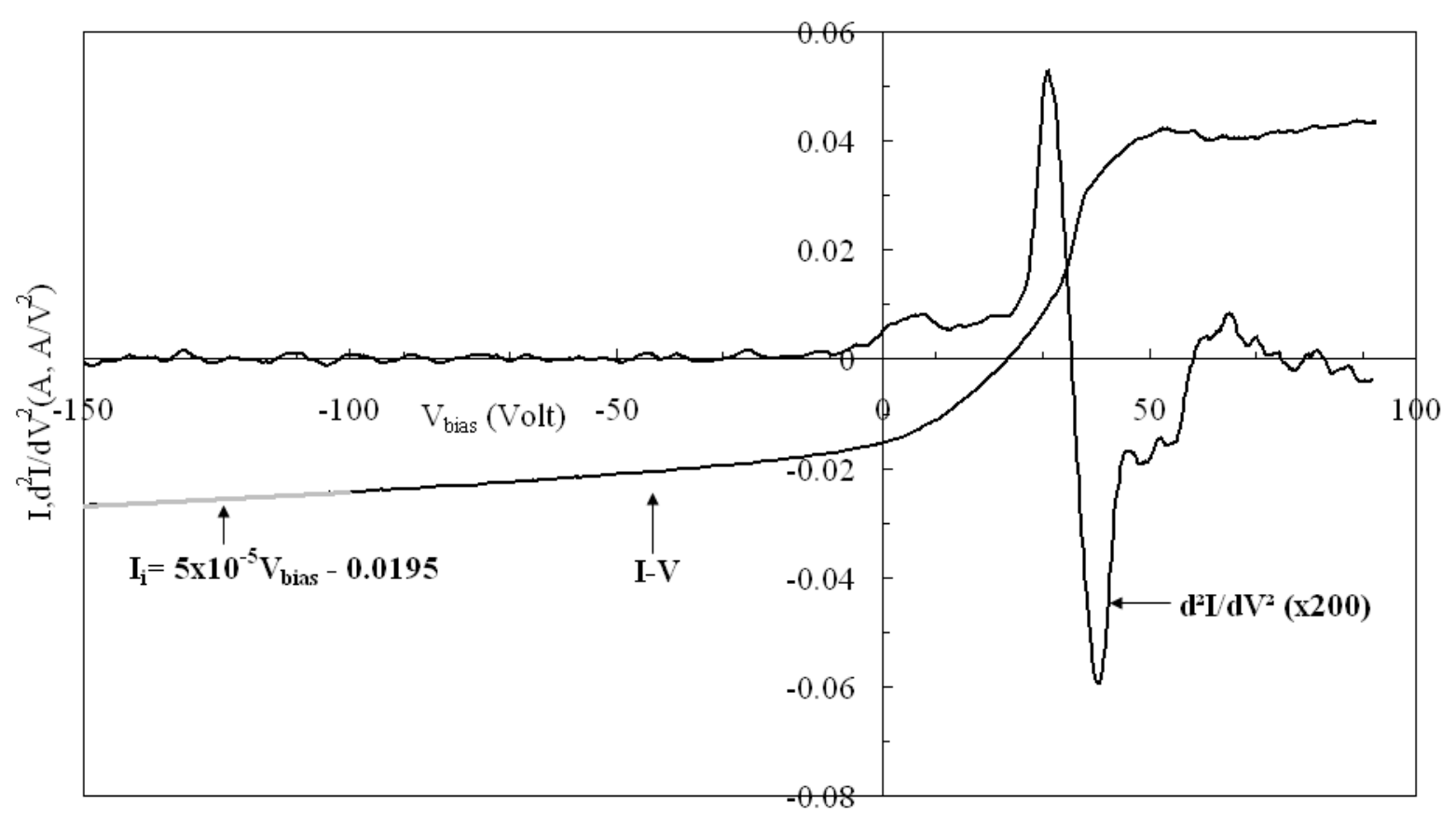

Fig.1 I-V and $d^{2} I / d V^{2}$ versus $V$ bias voltage of the probe. The second derivative values have been multiply by 200 . 


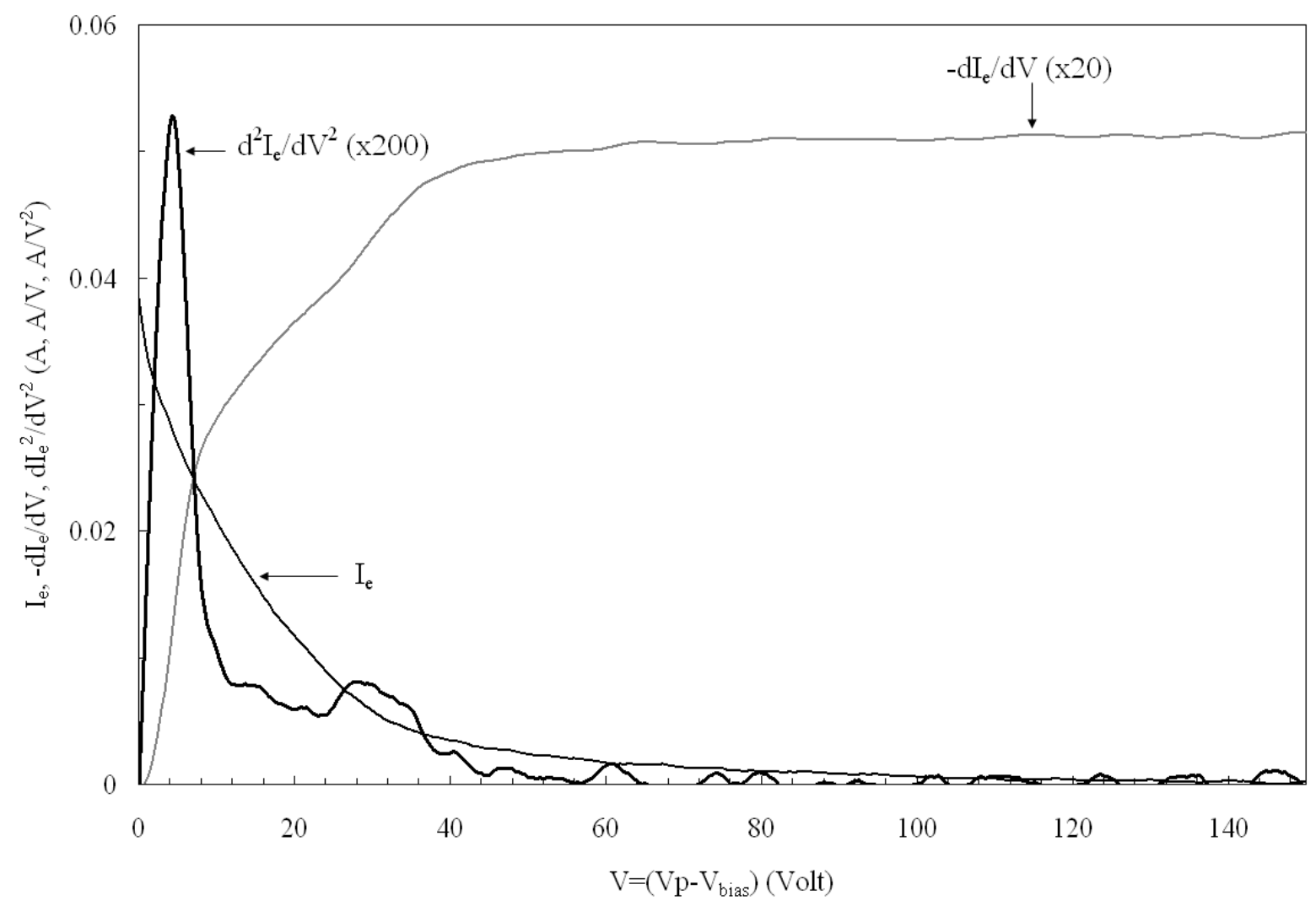

Fig.2 $I_{e},-d I_{e} / d v$ and $d^{2} I_{e} / d V^{2}$ versus $V$. The first and second derivative values have been multiply by 20 and 200, respectively.

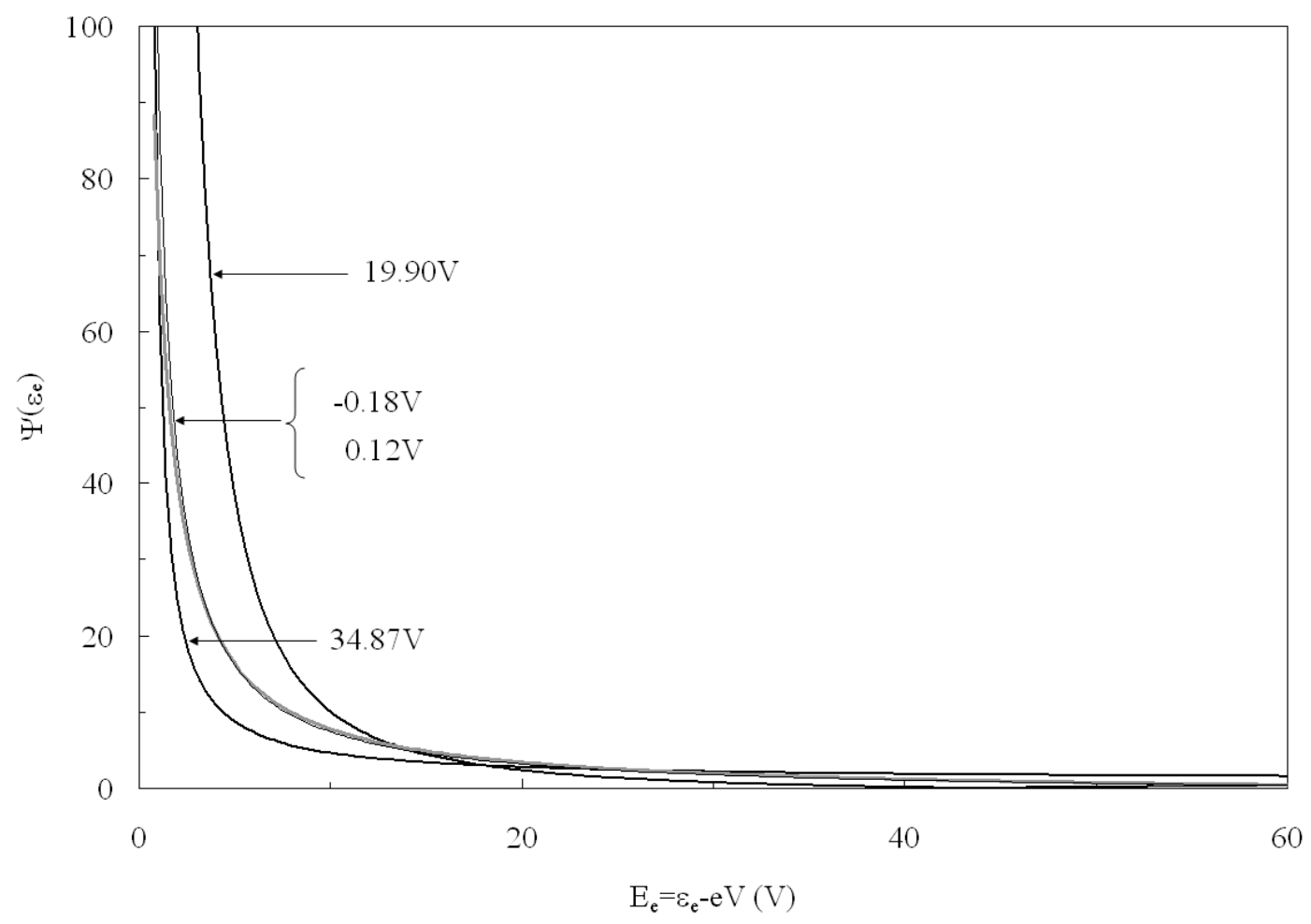


Fig.3 $\Psi\left(\varepsilon_{\mathrm{e}}\right)$ versus $\varepsilon_{\mathrm{e}}-\mathrm{eV}$.

The EEDF is determined using these $\Psi\left(\varepsilon_{\mathrm{e}}\right)$ values and EQ 4 and 6 . The numerical method proposed to determine the EEDF when we know $\Psi\left(\varepsilon_{\mathrm{e}}\right)$ is the following:

First we calculate an initial $\mathrm{EEDF} \mathrm{F}_{0}\left(\varepsilon_{\mathrm{e}}\right)$ from the I-V probe characteristic, assuming that the magnetic field $\mathrm{B}=0 \mathrm{~T}$ and using the Druyvesteyn equation. This correspond to the case where $\Psi\left(\varepsilon_{\mathrm{e}}\right)=0$ in EQ6 and is equivalent to consider that the electron diffusion through the sheath is not influenced by the magnetic field.

Second, we calculate the second derivative values using EQ6 and the initial EEDF $F_{0}\left(\varepsilon_{\mathrm{e}}\right)$ and $\Psi\left(\varepsilon_{\mathrm{e}}\right)$ previously calculated using EQ9 then we compare these second derivative values to the experimental ones (shown in Fig 2). So, the difference between the experimental and calculated derivatives is due to the magnetic field effect.

Third, we increment the former $\operatorname{EEDF}\left(\mathrm{F}_{0}\left(\varepsilon_{\mathrm{e}}\right)\right)$ using the difference between the two second derivatives, the experimental and the calculated and we determine a new $\operatorname{EEDF}\left(\mathrm{F}_{1}\left(\varepsilon_{\mathrm{e}}\right)\right)$ using,

$\varepsilon_{e}^{-1 / 2} F_{1}\left(\varepsilon_{e}\right)=\varepsilon_{e}^{-1 / 2} F_{0}\left(\varepsilon_{e}\right)+\alpha\left[\left(\frac{\partial^{2} j_{e}(V)}{\partial V^{2}}\right)_{\exp }-\left(\frac{\partial^{2} j_{e}(V)}{\partial V^{2}}\right)_{E Q 9}\right] . \quad$ EQ10

In this equation $\alpha$ is an increment used to adjust the EEDF. It is an arbitrary value generally ranging from 0 to 2 which is adjusted at each step of the calculation to obtain the good result. Fourth, using this new EEDF, we calculate the second derivative using EQ6 again and compare the calculated values with the experimental ones. If the values are different, we increment again the EEDF, changing the value of $\alpha$ in EQ10 until a perfect agreement between calculated and experimental values of the second derivative. The good agreement between these values corresponds to the good $\operatorname{EEDF} F_{1}\left(\varepsilon_{\mathrm{e}}\right)$. It is worth noting that the increment of the EEDF can also be performed at constant $\alpha$ changing $\mathrm{F}_{0}\left(\varepsilon_{\mathrm{e}}\right)$ by the new EEDF in EQ10 and calculating a new $F_{1}\left(\varepsilon_{e}\right)$, until a perfect agreement between experimental and calculated second derivative using EQ6.

Fifth, we calculate the electron current $i_{e}$ versus $V$ corresponding to this EEDF $F_{1}\left(\varepsilon_{e}\right)$ using EQ4. Then we add the ion current to this value and compare the results to the experimental I$\mathrm{V}$ probe characteristic, to check the accuracy of these results.

Generally the convergence between the two second derivatives (experimental and calculated one is obtained after two or three increments i.e. adjusting 2 or 3 times the value of $\alpha$. 
Fig 4 shows the agreement obtained between the experimental and calculated second derivatives in the case of the example under investigation. Two second derivatives calculated using $\alpha=0$ and $\alpha=1.2$ in EQ10 are compared with experimental values. $\alpha=0$ corresponds to the second derivative calculated using the Druyvesteyn equation $\left(\mathrm{F}_{0}\left(\varepsilon_{\mathrm{e}}\right)\right)$. When $\alpha=1.2$ in EQ10, the second derivative calculated using $\mathrm{F}_{1}\left(\varepsilon_{\mathrm{e}}\right)$ in EQ6 fits well with the experimental values.

To check the accuracy of these results (fifth step), we have calculated the electron current collected using EQ4, the EEDF corresponding to $\alpha=1.2$ and the total current. Results are shown on Fig5. A good agreement is observed between experiments and calculations. This shows the accuracy of the method.

As previously explained, we have successfully tested the method in the case of different I-V probe characteristics obtained in different magnetic field with intensity ranging from 0T to $1.2 \mathrm{~T}$ in the same experimental set-up.

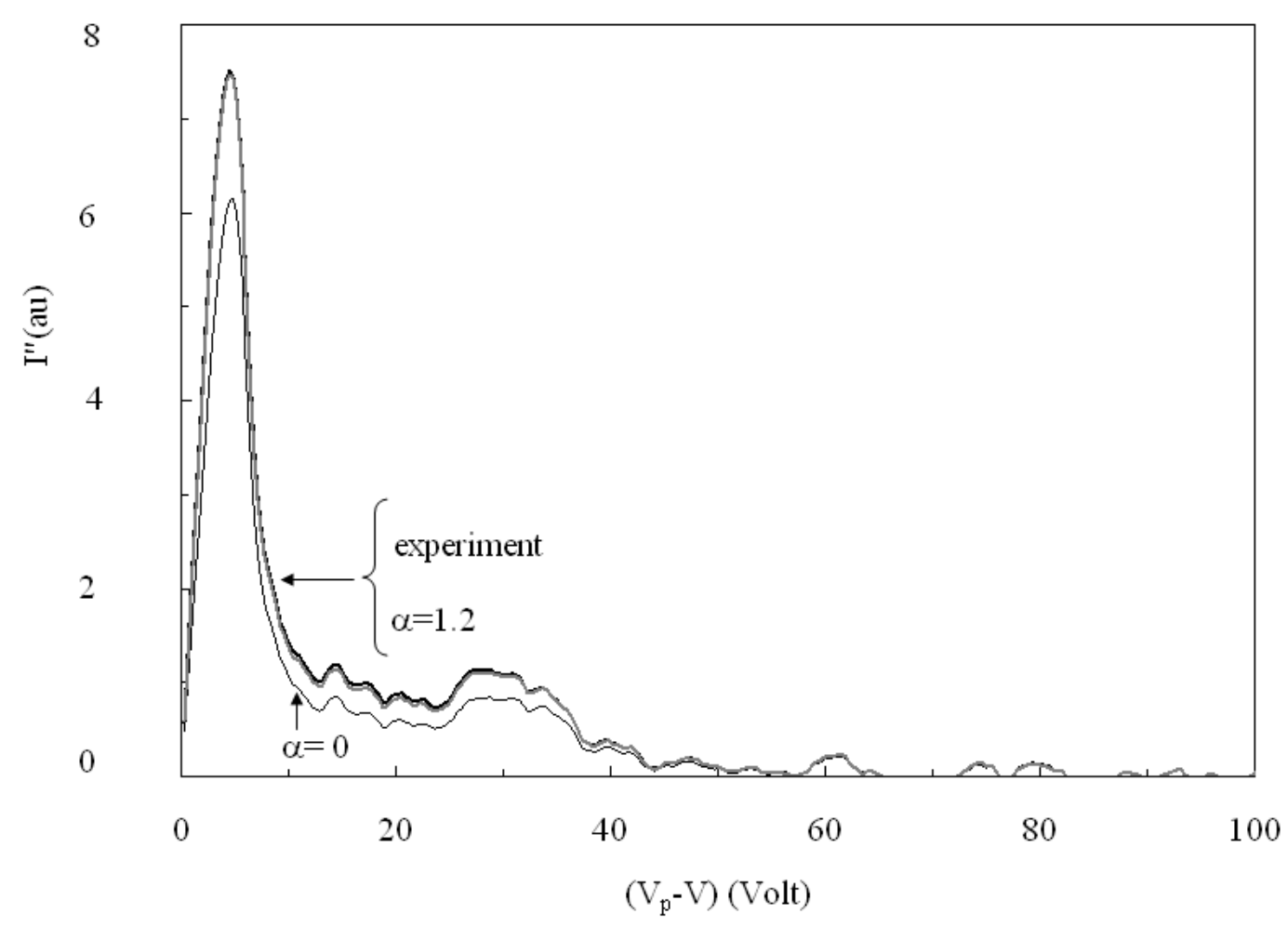

Fig.4 Second derivative calculated using EQ6, when $\alpha=0$ and $\alpha=1.2$ in EQ10, respectively; Comparison with experimental results. 


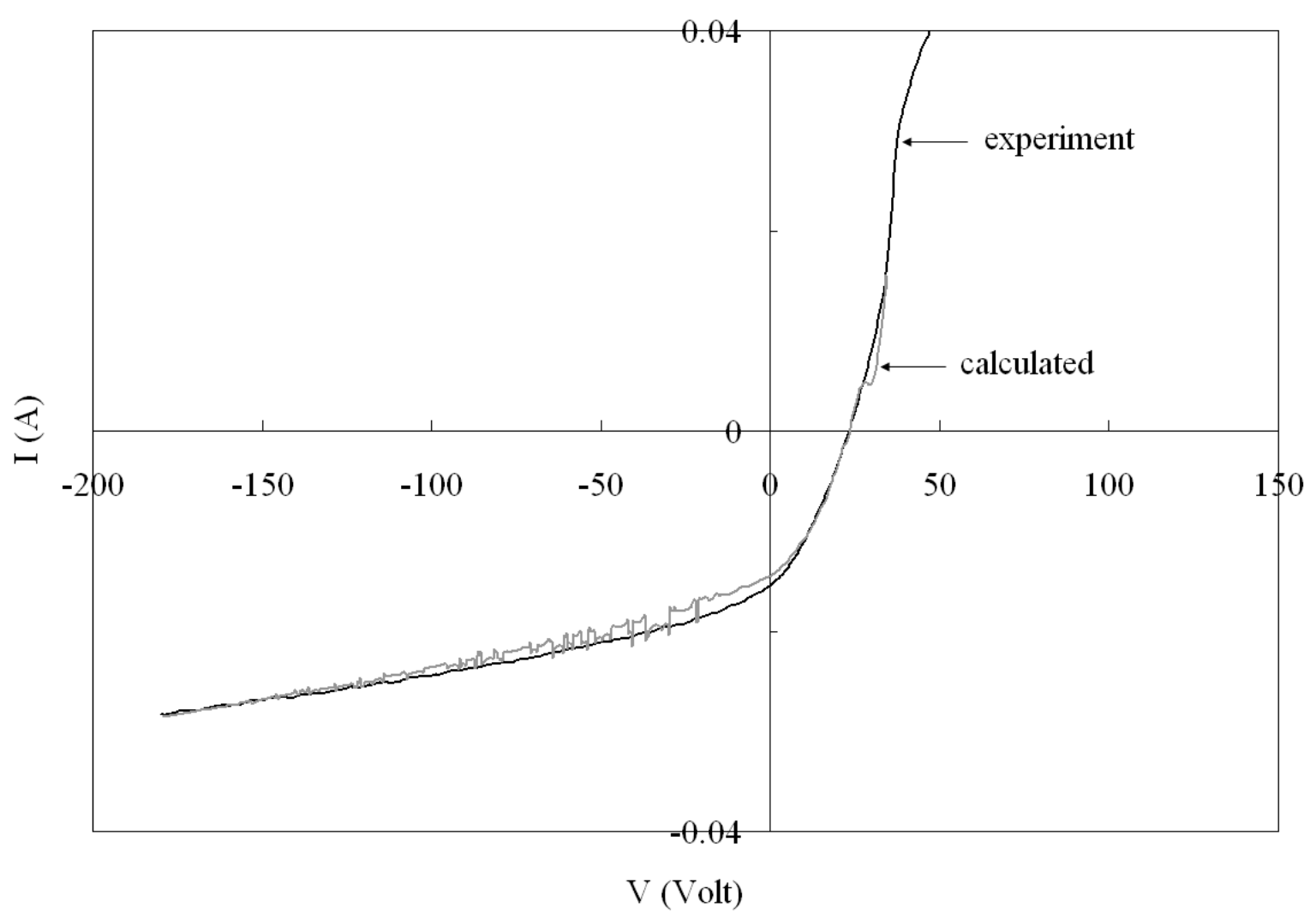

Fig.5 I-V probe characteristic: Comparison between experimental and calculated values.

We have compared our method to the method proposed in literature by Arslanbekov et al [4] or Popov et al [5], in the case of the same experiment.

Resolving the kinetic equation for electrons, these authors obtain the following equation of diffusion parameter,

$$
\Psi\left(\varepsilon_{e}, V\right)=\frac{1}{\gamma \lambda\left(\varepsilon_{e}\right)} \int_{a}^{\infty}\left(\frac{a}{r}\right)^{n} \frac{D\left(\varepsilon_{e}\right)}{D\left(\varepsilon_{e}-e \phi(r)\right)} d r
$$

Where "a" is the probe radius and $\gamma$ is a geometrical factor ranging from $4 / 3$ to 0.71 with increasing $a / \lambda$ ratio. $n=1$ in the case of a cylinder and 2 in the case of a sphere.

In this equation $\Psi\left(\varepsilon_{\mathrm{e}}\right)$ depends on the electron mean free path and on the diffusion coefficient. Both depend on the electron energy which changes through the sheath produced around the probe. So, $\gamma$ also is expected to change through the sheath. Moreover, the diffusion parameter depends also on the probe potential profile $\left(\Phi\left(\varepsilon_{\mathrm{e}}\right)\right)$ through the sheath.

As previously explained, in the presence of a magnetic field, the coefficient of diffusion becomes a tensor with a component parallel and the other perpendicular to the magnetic field and the kinetic equation for the EEDF has also the form of an anisotropic diffusion equation. When the probe is located parallel to the magnetic field, assuming a thin sheath with constant 
diffusion coefficient and electron mean free path through the sheath and a potential profile increasing linearly with $r$, when $b<\rho a$ the diffusion parameter is written $[4,5]$,

$\Psi\left(\varepsilon_{e}\right)=\frac{S \rho}{8 \pi \sqrt{\left(\left|a^{2}-b^{\prime 2}\right|\right) \lambda\left(\varepsilon_{e}\right) \gamma}}\left(\pi-2 \arctan \left(\frac{b^{\prime}}{\sqrt{\left(\left|a^{2}-b^{\prime 2}\right|\right)}}\right)\right)$

Where $\mathrm{S}$ is the probe area, $\rho=\left[1+\left(\frac{\lambda\left(\varepsilon_{e}\right)}{R_{L}\left(\varepsilon_{e}\right)}\right)^{2}\right]^{1 / 2}, \mathrm{~b}^{\prime}=\mathrm{b} / \rho, \mathrm{b}=\mathrm{L} / 2, \mathrm{~L}$ is the probe length.

Assuming $\mathrm{R}_{1}<<\lambda\left(\varepsilon_{\mathrm{e}}\right)$, Popov et al [5] simplify EQ12 which becomes,

$\Psi\left(\varepsilon_{e}\right)=\frac{L \pi}{4 R_{L}\left(\varepsilon_{e}\right) \gamma}$

Conversely, in the case where $\mathrm{b}>\rho \mathrm{a}[4,5]$,

$\Psi\left(\varepsilon_{e}\right)=\frac{S \rho}{8 \pi \sqrt{\left(\left|a^{2}-b^{\prime 2}\right|\right) \lambda\left(\varepsilon_{e}\right) \gamma}} \ln \left(\frac{\sigma+1}{\sigma-1}\right)$

With $\sigma=\frac{b^{\prime}}{\sqrt{\left(\left|a^{2}-b^{\prime 2}\right|\right)}}$.

In hydrogen plasma, the electron mean free path is calculated using the e-N collision cross section given by Yoon et al [10]. The electron mean free path first decreases until about $0.04 \mathrm{~m}$ at electron energy lower than $3 \mathrm{eV}$ and increases up to $1 \mathrm{~m}$ at electron energy of about $200 \mathrm{eV}$. Under these conditions $\mathrm{b}<\rho \mathrm{a}$ and EQ12 or EQ13 instead of EQ14 must be used. Fig6 compares the diffusion parameter versus electron energy calculated using our method and EQ12 and EQ13. EQ12 and 13 give the same results which are overestimated compared to ours. A better adjustment between our method and the theory of Arslanbekov et al (or Popov et al) is obtained when the diffusion parameter values calculated using EQ13 are divided by 10 as shown on Fig6. 


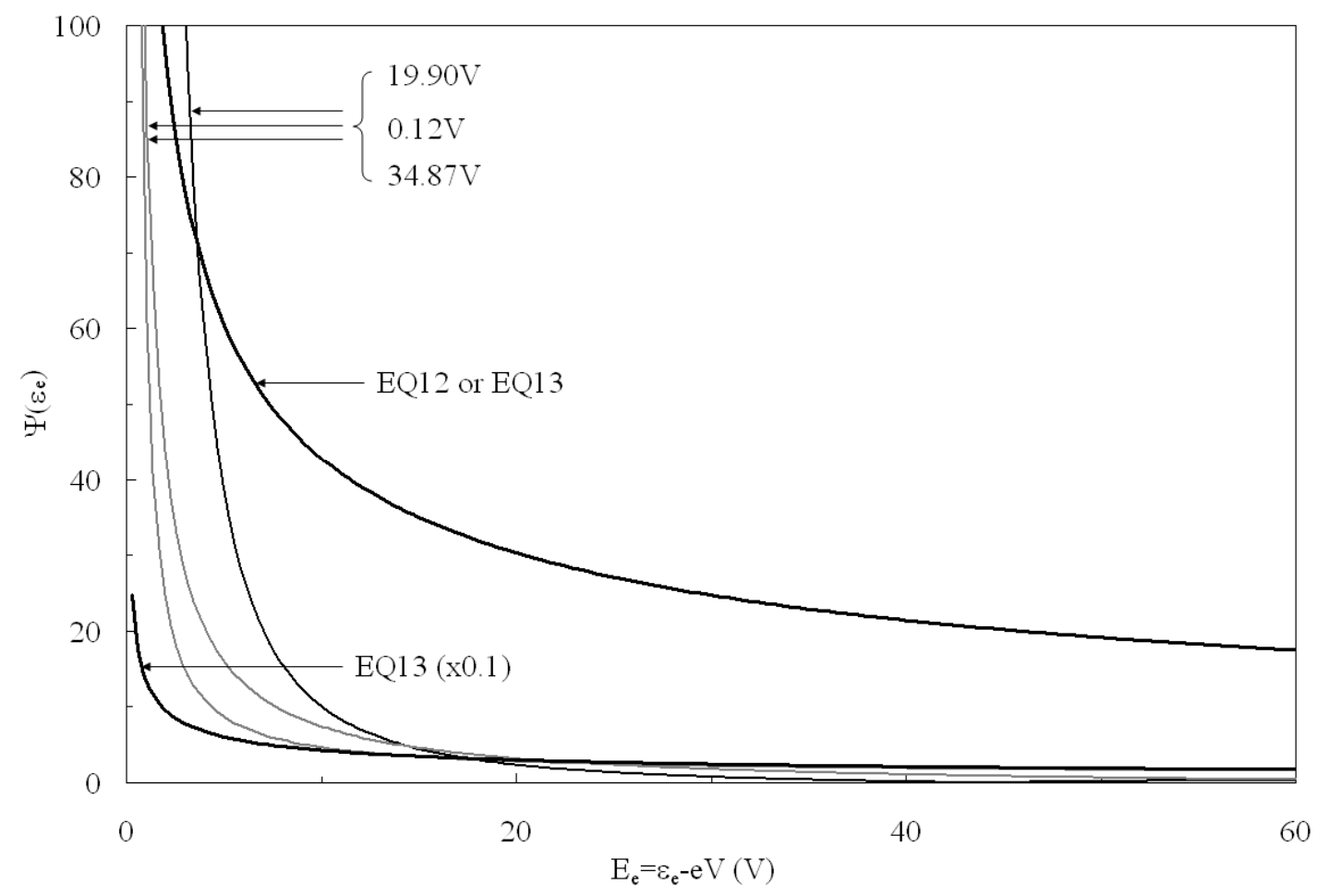

Fig.6 Comparison between the values of $\Psi\left(\varepsilon_{\mathrm{e}}\right)$ versus $\varepsilon_{\mathrm{e}}$ calculated using our method (for different value of V) and using the theory of Arslanbekov et al [4] EQ12 or EQ13. We report also values corresponding to EQ13 divided by 10 (EQ13x0.1).

We have compared the EEDF calculated using Eq13 multiplying $\Psi\left(\varepsilon_{\mathrm{e}}\right)$ by a factor ranging from 1 to 0.01 with the EEDF calculated using our method and the EEDF calculated assuming $\mathrm{B}=0 \mathrm{~T}$ (using the Druyvesteyn equation). Then, we have calculated the I-V probe characteristic using these EEDF in EQ4 and compare the results to the experimental I-V probe characteristic. Results are shown on Fig7 and Fig8. 


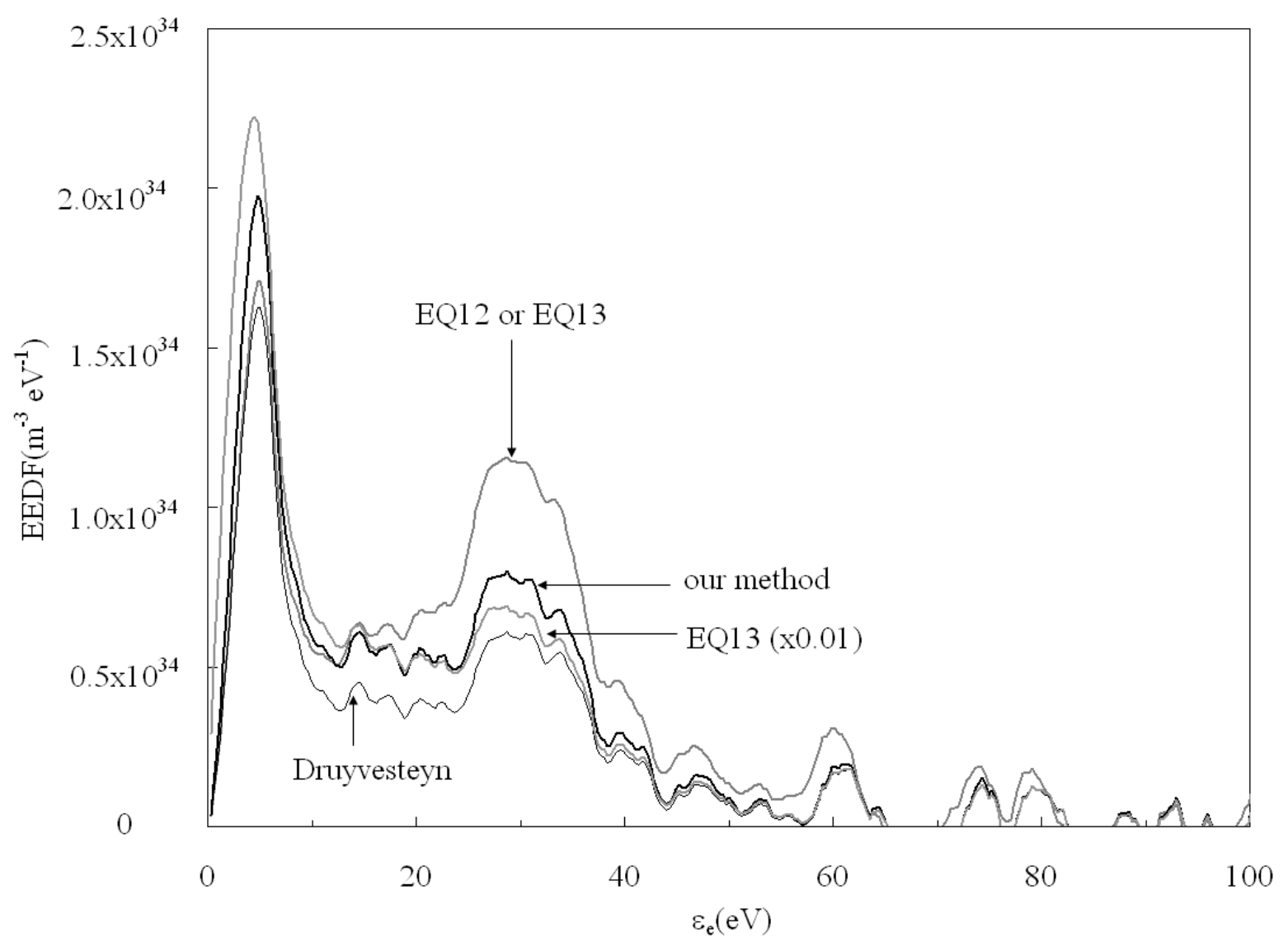

Fig.7 Comparison of EEDF versus electron energy calculated using the theory of Popov et al [5] (EQ13) then multiplying $\Psi\left(\varepsilon_{\mathrm{e}}\right)$ by (1 and 0.01) and using our method. Also is added for comparison the EEDF calculated using the Druyvesteyn Equation $(B=0 T)$. 


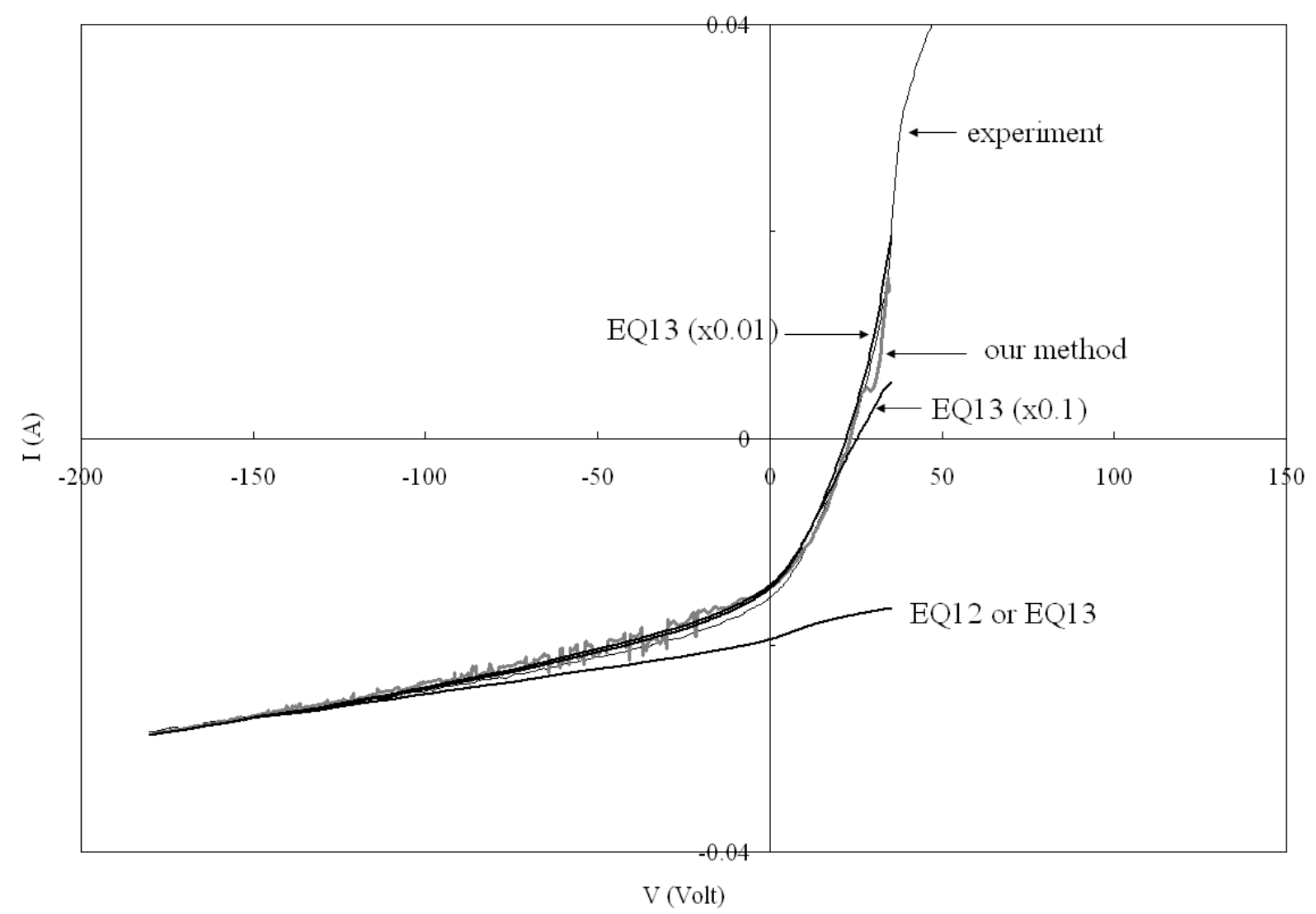

Fig.8 Comparison of experimental I-V probe characteristic with the calculated ones using EQ4.

As previously shown, the solutions of EQ13 (or EQ12) lead to overestimated values of $\Psi\left(\varepsilon_{\mathrm{e}}\right)$ which cannot be used to determine the right value of the EEDF. The diffusion parameter overestimates the effect of the magnetic field on the decrease of the electron current collected by the probe (see Fig8).

A good correlation between results is observed when the diffusion parameter values obtained using EQ13 are $\Psi\left(\varepsilon_{\mathrm{e}}\right) / 100$. In this case, a good fit between experimental and theoretical I-V probe characteristics is observed in Fig8. It is worth noting that even for lower $\Psi\left(\varepsilon_{\mathrm{e}}\right)$ values and until $\Psi\left(\varepsilon_{\mathrm{e}}\right)=0$, the results are well correlated. In this last case $\left(\Psi\left(\varepsilon_{\mathrm{e}}\right)=0\right)$, the EEDF is equivalent to the EEDF calculated assuming $B=0 T$, which is obviously wrong. So the method reported by Popov et al [5] (when it is corrected), can be used to determine the larger values of $\Psi\left(\varepsilon_{\mathrm{e}}\right)$ which gives reliable results for calculated I-V probe characteristics. The right EEDF is between the EEDF calculated using these larger values of $\Psi\left(\varepsilon_{\mathrm{e}}\right)$ and $\Psi\left(\varepsilon_{\mathrm{e}}\right)=0$. In contrast our method gives directly the exact value of $\Psi\left(\varepsilon_{\mathrm{e}}\right)$ (EQ9) then the right EEDF (see Fig7 and 8).

The different hypothesis used to obtain EQ12-14, as the electron mean free path, Larmor radius, electron diffusion coefficient, sheath potential profile or the probe orientation within 
the magnetic field are probably somehow erroneous or too approximate to provide good diffusion parameter values. According to literature $[11,12,13]$ the electron mean free path depends on the magnetic field intensity when this one is significant and losses its meaning as a characteristic parameter. The electron-neutral frequency becomes an efficient electronneutral frequency depending on $\rho$ and the Larmor radius becomes the effective mean free path. So, the diffusion coefficient is changed even in the direction parallel to the probe.

The new method reported in this work does not depend on such hypothesis and does not need the knowledge of the magnetic field intensity or of the probe orientation in this field so it is more convenient to determine the diffusion parameter and the EEDF in magnetized plasma.

Fig9 and Fig10 show other results obtained in the case of a $\mathrm{H}_{2}$ magnetized plasma working at a frequency of $2.45 \mathrm{GHz}, \mathrm{P}=0.38 \mathrm{~Pa}$ and at larger magnetic field $\mathrm{B}=0.12 \mathrm{~T}$. In Fig9, the difference between EEDF values obtained neglecting the magnetic field effect (Druyvesteyn) and the corrected ones is mainly observed at low electron energy when the Larmor radius is low. Experimental and calculated I-V probe characteristics are also in good agreement, as shown in Fig10.

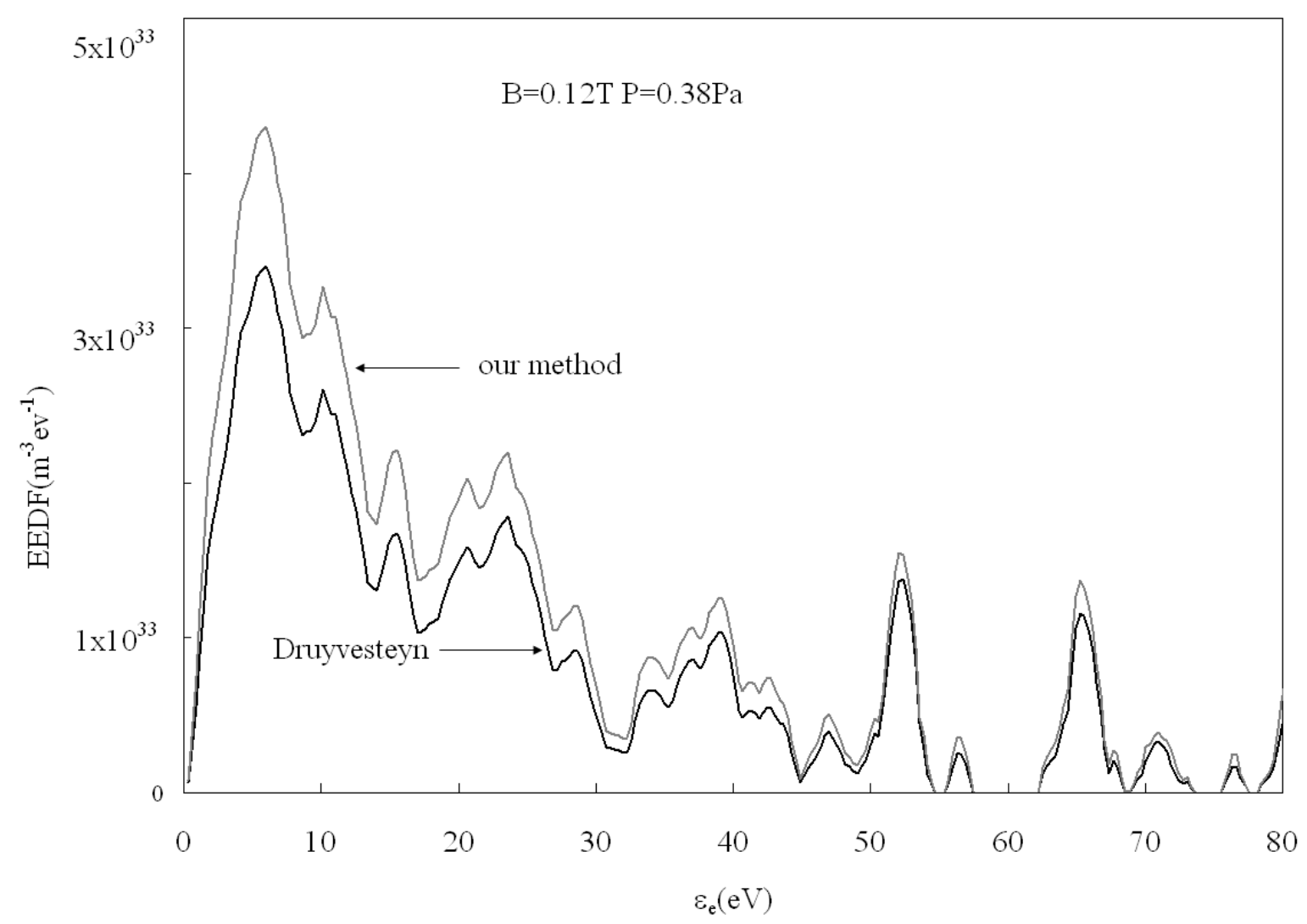

Fig.9 EEDF calculated using our method and neglecting the magnetic field effect (Druyvesteyn equation). 


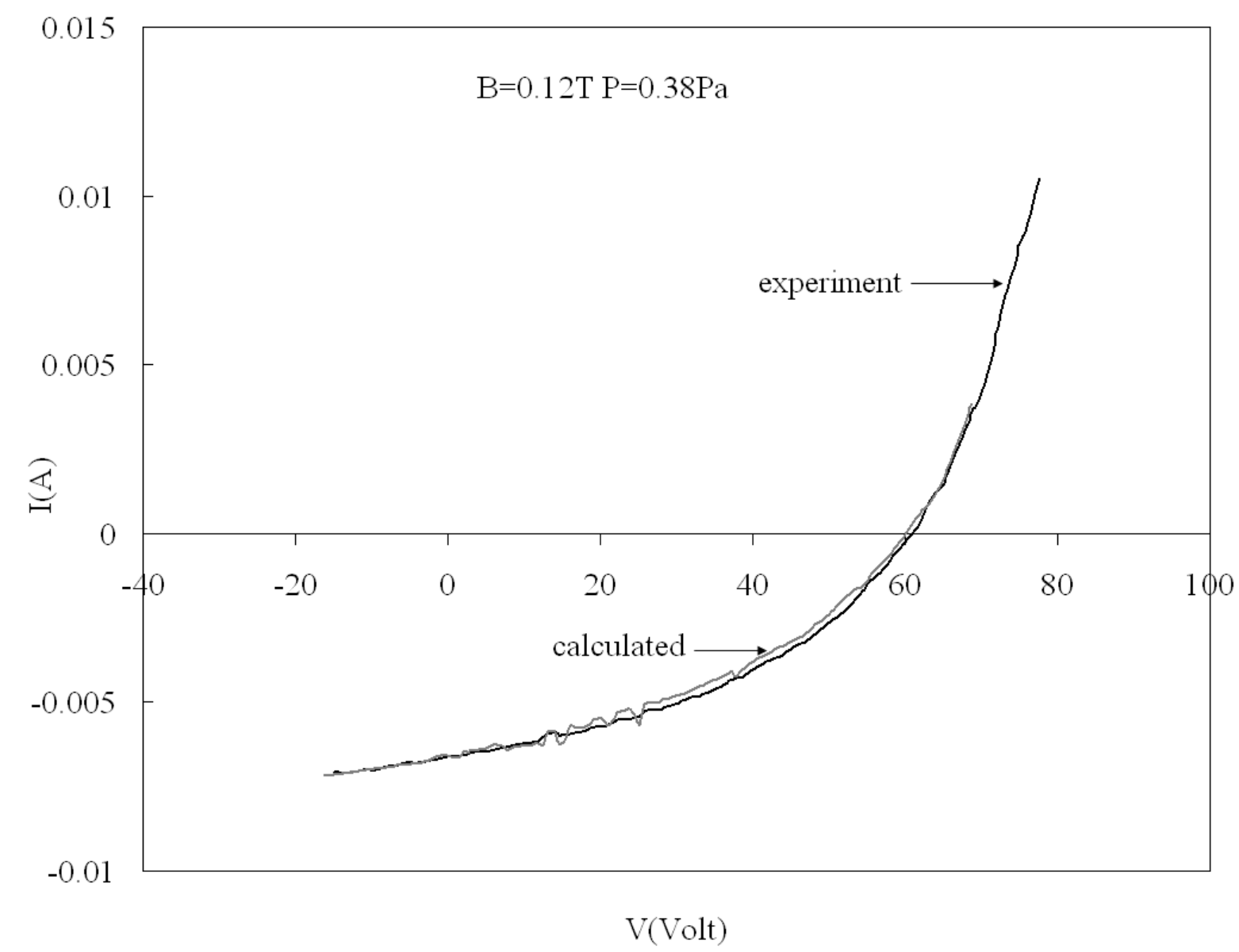

Fig.10 Comparison between experimental and calculated I-V probe characteristics; the calculated one is obtained using the EEDF calculated using our method and shown on Fig9.

\section{Conclusion.}

In this article a new method is reported to determine the Electron Energy Distribution Function (EEDF) in magnetized plasma, by means of a Langmuir single probe. This method is quite reliable whatever the probe orientation, diffusion coefficient, potential profile and electron mean free path through the sheath formed around the Langmuir probe. Moreover, the knowledge of the magnetic field intensity and profile within the reactor is not necessary. The diffusion parameter values are directly calculated from experimental I-V probe characteristics. So we do not need any model and assumptions to determine these values. We detail the method used to determine the EEDF calculating the diffusion parameter values from the I-V probe characteristics. It is tested for measurements performed in hydrogen magnetized microwave plasma and the results are compared to those obtained using the method reported in literature by Arslanbekov et al [4] or Popov et al [5]. We show that the method in [4] and 
[5] overestimates the diffusion parameter value and need an additional correction factor before the good results are obtained. This is probably either due to the different hypothesis used to simplify the calculation of the diffusion parameter or to the probe orientation which is not well defined in the magnetic field. Conversely, our method directly gives the diffusion parameters values from experimental I-V probe characteristics and the corresponding EEDF and is more convenient than the previous method reported in literature. The diffusion parameter is directly calculated using local experimental data and does not depend on hypothesis or on errors made measuring the magnetic field intensity within the reactor. So, it can be efficient to study electron kinetic in magnetized plasma like ECR or Helicon, where the magnetic field can be locally disturbed within the reactor.

It is worth noting that whatever the method used, the measured EEDF corresponds to the EEDF of the plasma bulk only if the electron energy relaxation length is much larger than the probe disturbed length. Otherwise, the EEDF can be changed in the disturbed region around the probe $[4,5,6]$ and is not representative of the plasma bulk.

\section{References.}

[1]. Microwave Excited Plasmas, Plasma Technology 4, Edited by M. Moisan, J. Pelletier, Elsevier, Amsterdam p181-212 (1992).

[2]. D. Bohm. Characteristics of Electrical Discharges in Magnetic Fields ed A. Guthrie and R.K. Wakerling (New York: McGraw-Hill) (1949)

[3]. J.D. Swift, Proc. Phys. Soc. 79 697-701 (1962).

[4]. R.R. Arslanbekov, N.A. Khromov, A. Kudryavtsev, Plasma Source Sci. Technol. 3 528538 (1994).

[5]. T.K. Popov, P. Ivanova, M. Dimitrova, J. Kovačič, T. Gyergyek, M. Čerček, Plasma Sources Sci. Technol. 21025004 (2012)(10pp).

[6]. V.I. Demidov, R. Armstrong, A. Fredriksen, Contrib. Plasma. Phys. 38 (1998) S, 1-6.

[7]. O D Cortázar, J Komppula, O Tarvainen, A Megía-Macías,A Vizcaíno-de-Julián and H Koivisto. Plasma Sources Sci. Technol. 22 (2013) 015026 (9pp).

[8]. J.L. Jauberteau, I. Jauberteau, Meas. Sci. Technol. 18, 1235-1249 (2007).

[9]. F. Jauberteau, J.L. Jauberteau, Applied Mathematics and Computation 215 2283-2297 (2009).

[10]. J.S. Yoon et al, J. Phys. Chem. Ref Data 372 (2008).

[11]. A. Fridman, Plasma chemistry, Cambridge University Press, Cambridge p 150 (2008). 
[12]. Microwave Excited Plasmas, Plasma technology, 4, M Moisan, J Pelletier editors, Ed Elsevier p 182 (1992).

[13]. Physics of Radio-Frequency Plasmas P. Chabert, N. Braithwaite, Ed Cambridge University Press p 294-295 (2011). 